\title{
Le temps de la mobilisation est (re)venu!
}

\author{
Jean-Félix Savarya, Jean Martin ${ }^{b}$ \\ a Secrétaire général du GREA; ${ }^{b}$ Dr méd., ancien médecin cantonal (Vaud)
}

Fin 2018, le Conseil fédéral a pris la décision de supprimer les trois commissions fédérales traitant d'addiction, à savoir de drogues, de tabac et d'alcool. Après avoir refusé d'en créer une sur les jeux d'argent, il choisit de tout fusionner dans le cadre d'une unique «Commission fédérale pour les questions liées à l'addiction et pour la prévention des maladies non transmissibles». Erreur funeste, à plus d'un titre, mais qui doit sonner le réveil chez les professionnels du domaine.

Erreur d'appréciation d'abord, car le Conseil fédéral confond stratégie à long terme et pragmatisme à court terme. Avec raison, nous avons adopté en 2017 une nouvelle "Stratégie addiction", pour traiter ces questions de manière coordonnée. L'alcool, l'héroïne et le cannabis ne sont pas devenus les mêmes produits pour autant. Ils demandent chacun des réflexions spécifiques, selon leur dangerosité, leur ancrage social, leur poids économique et culturel. Si, à long terme, l'objectif est de disposer d'une véritable politique des addictions, il faut reconnaître que les problèmes restent encore très différents sur le terrain. Le tabac fumé tue, alors que l'alcool produit d'innombrables difficultés personnelles et sociales et que la prohibition des drogues amène une foule d'autres problèmes, entre stigmatisation et punition.

Chaque marché et chaque produit a ses enjeux propres: se débarrasser de la combustion pour la nicotine, réduire les risques de produits dangereux confiés aux mafias pour les drogues et encadrer au mieux la consommation pour l'alcool. Vouloir gommer ces différences sous prétexte qu'il s'agit à chaque fois de produits psychotropes ne peut nous aider à avancer adéquatement.

Mais il y a plus grave. Avide de synergies et d'économies, le Conseil fédéral propose d'arrimer les addictions aux maladies non transmissibles (MNT). Les liens de causalité entre consommation de psychotropes et maladies (cancers, AVC, etc.) l'incitent à faire glisser le tout dans une même problématique sanitaire. A vouloir trop prendre de hauteur, la finesse de la réalité et le sens des actions se perd. Il est louable de jeter des ponts et d'insuffler l'interdisciplinarité des addictions dans le domaine des MNT. Mais parle-t-on vraiment de la même chose? Alors que les MNT pèsent de tout leur poids sur les coûts de la santé, les addictions plombent elles notre vie en communauté. Si, pour traiter ces dernières, il s'agit bien d'une réponse sanitaire classique, les politiques en matière d'addiction relèvent elles d'une autre logique, celle du travail en réseau et de l'approche communautaire. La gestion des MNT doit, bien entendu, aller elle aussi au-delà du médico-sanitaire. Alimentation, mouvement, équité, travail, représentent des enjeux majeurs que nous devrons bien un jour considérer sous l'angle de la promotion de la santé. Aujourd'hui, nous en sommes encore loin et la prévention ne constitue qu'une part mineure des actions en la matière.

\section{Des logiques différentes}

La Suisse a su trouver une politique originale sur quatre piliers en matière de drogues illégales. Bien que fortement médiatisées, les politiques publiques en la matière restent cependant modestes et font beaucoup avec peu de moyens. Des structures à bas seuil d'accès permettent d'offrir des prestations en dehors du système traditionnel de santé, en réussissant à contourner les obstacles pratiques pour cette population (stigmatisation, prohibition). Des collaborations parfois audacieuses entre sécurité, ordre public, social et santé ont vu le jour, en faisant un domaine tout à fait à part, à l'intersection de diverses politiques sectorielles.

Les maladies, quant à elles, sont une aire de spécialistes où les meilleurs traitements se doivent d'être mis à disposition de la population dans les meilleures conditions possibles. Il s'agit là d'une logique technique basée sur la maîtrise de processus validés par la science médicale. Si des liens existent entre les deux champs, les MNT et les addictions n'ont somme toute que peu de choses en commun.

\section{A la recherche du leadership perdu}

Si la Confédération a longtemps été source d'inspiration pour les cantons en matière d'addiction, le vent a tourné depuis plusieurs années. Les coupes annuelles dans le budget fédéral laissent les différentes communautés du pays face à leurs difficultés et leurs problèmes. La question du deal de rue n'est pas résolue. Villes et cantons 
ont mendié au Parlement, sans être entendus, des mesures minimales sur l'alcool, afin notamment d'apaiser les nuits urbaines et les services d'urgence surchargés. Dans un même temps, la discussion sur une nouvelle loi sur les produits du tabac s'est ouverte et risque manifestement de manquer sa cible.

Animé par une vision moderne, un certain volontarisme marquait notre pays dans les années 1990. Avec un Conseil fédéral courageux et audacieux, la Confédération avait su répondre de manière innovante à des problèmes complexes. La Suisse avait réussi à se forger une réputation grâce à sa politique des quatre piliers. Cette époque est bel et bien révolue. Néanmoins, nous nous empêtrons à présent dans les contradictions morales propres à tout discours sur les drogues. Il semble que le Conseil fédéral regarde ailleurs, préoccupé par d'autres sujets à son avis plus importants. Les déboires de la LAMal n'incitent pas à partir à l'aventure et, sur le terrain, les dommages sont moins visibles avec la fin des scènes ouvertes.

Cependant, les problèmes restent entiers, avec plus de 9000 décès par année dus au tabagisme et $15 \%$ des maladies qui sont liées à l'alcool. Si la mortalité a chuté pour les usagers de drogues injectables, les défis restent là aussi nombreux. Notamment, le deal de rue pourrit les débats publics dans les villes romandes, et de nouvelles formes de consommation problématique voient le jour, telles que l'augmentation de l'usage de la cocaïne. Le Conseil fédéral n'entend pas les préoccupations de la population à cet égard, et se réfugie derrière un parlement qui, sur ces sujets, a toujours privilégié les intérêts économiques aux légitimes préoccupations de santé publique et aux obligations de respect des droits humains.

\section{Et demain?}

L'abandon des commissions fédérales, bastions d'une réflexion indépendante au niveau national, porte un coup grave au domaine. Il faut se rappeler qu'en matière de politique drogues, les acteurs de terrain ont toujours tiré vers l'avant les autorités. En 1986, alors que la ville de Berne autorise le premier espace de consommation à moindres risques, le gouvernement s'y oppose. Ce n'est que cinq ans plus tard, sous la pression d'autres villes, qu'une nouvelle stratégie sera développée autour du concept de la réduction des risques. Au niveau local, des compromis ont pu être trouvés, plus proches des vrais problèmes, tels qu'ils se posent pour la population et les professionnels de santé, mais aussi de la sécurité, du social et de l'éducation. Le régime de nuit pour la vente d'alcool à Genève ou l'interdiction de la fumée passive au Tessin n'ont pas été inventés par la Confédération, mais par des acteurs cantonaux.

Les avancées en matière d'addiction ne peuvent ainsi pas être l'œuvre de la Confédération seule par une politique top-down. Les alliances au niveau local permettent de trouver de nouvelles idées et pratiques. Ensuite, si une mobilisation effective se produit, il est possible de changer les choses et la Confédération peut alors devenir une alliée. Elle ne saurait prendre seule l'initiative, principe de subsidiarité oblige. Par contre, une fois le diesel fédéral lancé, il peut produire des effets importants.

Aujourd'hui, le Conseil fédéral ne semble plus vouloir se préoccuper des addictions. Dommage. Mais il existe d'autres acteurs qui peuvent jouer un rôle important pour le changement. C'est un nouveau leadership que nous devons construire. Il ne s'agit pas pour autant de tourner le dos au chef d'orchestre des décennies passées. Les administrations fédérales restent des lieux où le savoir et l'expertise existent et d'où ils peuvent être diffusés et valorisés, grâce à des professionnels compétents et engagés.

La réduction des commissions fédérales en matière d'addiction ne marque donc pas la fin du chemin. La société civile doit maintenant se mobiliser, en forgeant de larges alliances pour imaginer et cimenter les idées de demain. Nous avons connu une époque où il y avait des moyens et une politique fédérale claire. Il est vrai, cette période succédait à une mobilisation importante des acteurs, suite aux scènes ouvertes et à la crise du VIH/SIDA. Aujourd'hui, avec la diversification des produits par Internet, la question des opioïdes, le deal de rue et la révolution qui s'annonce pour sortir de la prohibition, les sujets ne manquent pas. Il est temps de retrouver l'imagination et la souplesse nécessaires pour compenser la perte des commissions, et pour stimuler un renouveau du leadership nécessaire du Conseil fédéral.

\section{Crédit photo}

(c) Zdravinjo | Dreamstime.com
Correspondance: Jean-Félix Savary Secrétaire général GREA-Groupement Romand d'Etudes des Addictions Rue St-Pierre 3, CP 6319 CH-1002 Lausanne Tél. 0244202261 jf.savary[at]grea.ch

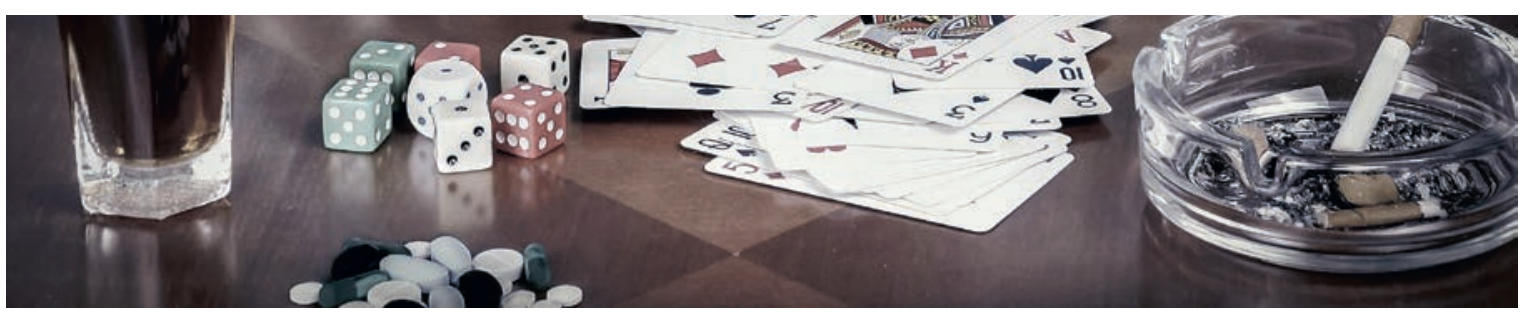

